\title{
Senolytic drugs target alveolar epithelial cell function and attenuate experimental lung fibrosis ex vivo
}

\author{
Mareike Lehmann ${ }^{1}$, Martina Korfei ${ }^{2}$, Kathrin Mutze ${ }^{1}$, Stephan Klee ${ }^{1}$, \\ Wioletta Skronska-Wasek ${ }^{1}$, Hani N. Alsafadi ${ }^{1}$, Chiharu Ota ${ }^{1}$, Rita Costa ${ }^{1}$, \\ Herbert B. Schiller ${ }^{1}$, Michael Lindner ${ }^{3}$, Darcy E. Wagner ${ }^{1}$, Andreas Günther ${ }^{2,4,5}$ \\ and Melanie Königshoff (i) ${ }^{1,6}$
}

\begin{abstract}
Affiliations: ${ }^{1}$ Comprehensive Pneumology Center (CPC), Helmholtz Zentrum München and University Hospital of the Ludwig Maximilians Universität, Member of the German Center for Lung Research (DZL), Munich, Germany. ${ }^{2}$ Dept of Internal Medicine, Universities of Giessen and Marburg Lung Center (UGMLC), Justus-Liebig-Universität Giessen, Member of the German Center for Lung Research (DZL), Giessen, Germany. ${ }^{3}$ Center for Thoracic Surgery, Asklepios Biobank for Lung Diseases, Comprehensive Pneumology Center, Asklepios Clinic Munich-Gauting, Munich, Germany. ${ }^{4}$ Agaplesion Lung Clinic Waldhof Elgershausen, Greifenstein, Germany. ${ }^{5}$ European IPF Network and European IPF Registry. ${ }^{6}$ Division of Pulmonary Sciences and Critical Care Medicine, Department of Medicine, University of Colorado, Denver, CO, USA.
\end{abstract}

Correspondence: Division of Pulmonary Sciences and Critical Care Medicine, Dept of Medicine, University of Colorado Denver, AMC, Research 2, 9th Flr, 12700 East 19th Ave, Aurora, C0 80045, USA.

E-mail: melanie.koenigshoffaucdenver.edu

@ERSpublications

Alveolar epithelial cell senescence occurs in IPF and senolytic treatment attenuates experimental lung fibrosis http://ow.ly/nFlz30bsmNm

Cite this article as: Lehmann M, Korfei M, Mutze K, et al. Senolytic drugs target alveolar epithelial cell function and attenuate experimental lung fibrosis ex vivo. Eur Respir J 2017; 50: 1602367 [https://doi.org/ 10.1183/13993003.02367-2016].

ABSTRACT Idiopathic pulmonary fibrosis (IPF) is a devastating lung disease with poor prognosis and limited therapeutic options. The incidence of IPF increases with age, and ageing-related mechanisms such as cellular senescence have been proposed as pathogenic drivers. The lung alveolar epithelium represents a major site of tissue injury in IPF and senescence of this cell population is probably detrimental to lung repair. However, the potential pathomechanisms of alveolar epithelial cell senescence and the impact of senolytic drugs on senescent lung cells and fibrosis remain unknown. Here we demonstrate that lung epithelial cells exhibit increased P16 and P21 expression as well as senescence-associated $\beta$-galactosidase activity in experimental and human lung fibrosis tissue and primary cells.

Primary fibrotic mouse alveolar epithelial type (AT)II cells secreted increased amounts of senescenceassociated secretory phenotype (SASP) factors in vitro, as analysed using quantitative PCR, mass spectrometry and ELISA. Importantly, pharmacological clearance of senescent cells by induction of apoptosis in fibrotic ATII cells or ex vivo three-dimensional lung tissue cultures reduced SASP factors and extracellular matrix markers, while increasing alveolar epithelial markers.

These data indicate that alveolar epithelial cell senescence contributes to lung fibrosis development and that senolytic drugs may be a viable therapeutic option for IPF.

Copyright OERS 2017 This ERJ Open article is open access and distributed under the terms of the Creative Commons Attribution Non-Commercial Licence 4.0. 


\section{Introduction}

Idiopathic pulmonary fibrosis (IPF) is a progressive interstitial lung disease with a median survival of 2-4 years [1]. Mechanisms involved in disease development and progression include repetitive injury to the lung epithelium, activation and proliferation of (myo)fibroblasts and altered production of extracellular matrix, together resulting in the destruction of lung architecture and function [1, 2]. Two drugs (pirfenidone and nintedanib) have been approved for the treatment of mild/moderate IPF [3, 4]; however, therapies halting or reversing disease progression are still lacking. Thus, there is a tremendous interest in deepening our understanding of the pathomechanism(s) underlying IPF in order to identify novel therapies.

The incidence of IPF increases with age and accumulating evidence strongly suggests ageing as a crucial contributor to IPF initiation and progression [5]. In support of ageing as one proposed driver of disease pathogenesis, normal and accelerated-aged mice are more susceptible to experimentally induced fibrosis $[6,7]$. A landmark paper in 2013 described nine hallmarks of ageing [8], and importantly, all nine hallmarks have been found to contribute to IPF pathogenesis, albeit to a variable degree [5]. Cellular senescence, representing one of these hallmarks, is characterised by stable cell cycle arrest accompanied by secretion of mediators, including pro-inflammatory cytokines and metalloproteinases, collectively termed the "senescence-associated secretory phenotype" (SASP) [9, 10]. While the detrimental effects of senescence are thought to be a result of stem or progenitor cell depletion or of the SASP components, senescence has also been described to be beneficial in tumour suppression and wound healing [10-12].

In the lung, as in other organs, the number of senescent cells increases with age [13] and cellular senescence has been linked to the pathogenesis of chronic lung diseases such as chronic obstructive pulmonary disease $[14,15]$ or IPF [16-20]. The contribution of senescent cells to disease onset and progression remain unclear. Some studies have suggested a link between increased senescence and fibrotic burden $[17,21,22]$, while others report that attenuation of lung fibrosis correlates with lung fibroblast senescence [23]. In addition to lung fibroblasts, evidence has emerged that alveolar epithelial cells can become senescent in IPF $[16,20,24]$. However, lung epithelial cell senescence and its potential pathogenic role in IPF remains largely unexplored. Here, we aimed to investigate whether senescence of this cell population is detrimental or beneficial to lung repair. We analysed cell senescence in lung tissue and in primary alveolar epithelial type (AT)II cells derived from human IPF and an experimental model of murine lung fibrosis. We demonstrate that depletion of senescent epithelial cells in vitro and ex vivo stabilises the epithelial cell phenotype and decreases fibrotic markers, indicating that senescence of alveolar epithelial cells may contribute to disease pathogenesis.

\section{Materials and methods}

\section{Senescence-associated $\boldsymbol{\beta}$-galactosidase staining}

Primary mouse ( $\mathrm{pm}$ ) ATII cells or three-dimensional lung tissue cultures (3D-LTCs) were prepared from PBS- or bleomycin-treated mice, as described previously [25] (online supplementary material) and cultured in multiwell plates. pmATII cells from PBS- and bleomycin-treated mice express high levels of prosurfactant protein (proSP)-C as well as the epithelial cell markers E-cadherin, cytokeratin (CK) and zona occludens (ZO)-1. Fibrotic ATII cells further exhibit co-staining of ZO-1 and proSP-C with $\alpha$-smooth muscle actin (figure 3a, online supplementary figure S4B and $[26,27]$ ). Cytochemical staining for senescence-associated (SA) $\beta$-galactosidase was performed using a staining kit (Cell Signaling Technology, Danvers, MA, USA), according to the manufacturer's instructions. Images were acquired using a Zeiss Axiovert40C microscope (Jena, Germany). The percentage of senescent cells was determined by counting of total and SA- $\beta$-galactosidase-positive cells in three random microscopic fields per condition (100× magnification).

\section{Flow cytometry-based detection of SA- $\beta$-galactosidase}

Flow cytometry-based detection of SA- $\beta$-galactosidase was performed as described previously [28]. Briefly, pmATII cells from PBS- and bleomycin-treated animals were incubated with bafilomycin A1 (100 nM;

This article has supplementary material available from erj.ersjournals.com

Received: Dec 022016 | Accepted after revision: April 212017

Support statement: This work was funded by grants from the German Center of Lung Research and a W2/W3 professorship award from the Helmholtz Association to M. Königshoff. D.E. Wagner is supported by a Whitaker International Scholar Fellowship and the Helmholtz Munich postdoctoral program. Funding information for this article has been deposited with the Crossref Funder Registry.

Conflict of interest: Disclosures can be found alongside this article at erj.ersjournals.com 
Enzo Life Sciences, Farmingdale, NY, USA) and $\mathrm{C}_{12}$ FDG (20 nM; Life Technologies, Carlsbad, CA, USA) for 1 and $2 \mathrm{~h}$, respectively, directly after isolation or at day 2 of culture. Cells were washed once and stained for allophycocyanin-conjugated epithelial cell adhesion molecule (EpCAM) antibody (118214; BioLegend, San Diego, CA, USA) for $20 \mathrm{~min}$ at room temperature, washed once and analysed using a fluorescence-activated cell sorter (LSRII; BD Bioscience, San Jose, CA, USA). Additional information can be found in the online supplementary material.

\section{Statistical analysis}

Data are presented as mean \pm SEM, from $\mathrm{n}$ separate experiments. Statistical significance of differences was evaluated using t-tests, paired t-tests or one-way ANOVA followed by a Newman-Keuls multiple comparison test, where appropriate. Correlation was evaluated using Pearson's test. Differences were considered to be statistically significant when $\mathrm{p}<0.05$. Additional information can be found in the online supplementary material.

\section{Results}

\section{Senescence marker expression is upregulated in the lung epithelium in IPF}

First, we aimed to investigate the occurrence of senescence in our IPF patient cohort. To this end, we analysed the gene expression of the senescence effector proteins cyclin dependent kinase inhibitor $(\mathrm{CDKN})$ 2A (P16) and CDKN1A (P21) in explanted lung tissue specimens of IPF or donor patients. P16 levels were significantly increased in lung homogenates of IPF patients as compared to donor lung homogenates (figure 1a; mean \pm SD change in threshold cycle $(\Delta \mathrm{Ct}$ ) donor $-1.91 \pm 0.74$ versus IPF $0.74 \pm 0.40$, $\mathrm{p}<0.01$ ), whereas $P 21$ levels remained unchanged. Our cohort matches results extracted from the Lung Genomics Research Consortium microarray data (GSE47460 and GPL4680) (online supplementary figure S1A). Furthermore, we found that P16 expression levels in IPF tissue negatively correlated with diffusing capacity of the lung for carbon monoxide (online supplementary figure S1B), indicating that patients with higher P16 levels had more severe disease. Furthermore, we observed increased P16 as well as P21 protein in whole-lung homogenates from IPF patients compared to donor lung tissue, as assessed using Western blotting (figure 1b).

To identify which cell types express phenotypic markers of senescence in IPF, we next performed immunohistochemical staining of P16 and P21 on IPF and donor lungs and found that IPF lungs exhibited intense nuclear and cytoplasmic staining for both P16 and P21 compared to age-matched donor lungs (figure 1c and d). Co-staining with epithelial cell marker proSP-C, KRT5 or KRT7 revealed that P16- and P21-positive cells were found in the alveolar epithelium of IPF lungs, largely in proSP-C $\mathrm{CRT}^{+}$ ATII cells (figure 1c; arrows), while no or only sporadic staining for P16 and P21 was observed in donor lungs (figure 1d; arrows). Furthermore, $\mathrm{KRT}^{+} \mathrm{KRT}^{+}$abnormal basal cells in areas of bronchiolisation exhibited positive staining for P16 and P21 (online supplementary figure S2A-C), while no or only weak staining was observed in mesenchymal cells (online supplementary figure S2A-D). In addition, P16 and P21 staining was observed in proSP-C ${ }^{-} \mathrm{KRT}^{-} \mathrm{KRT}^{+}$epithelial cells of IPF lungs (figure 1c; arrowheads). Notably, P21 (and P16) staining was also found in proSP-C ${ }^{+} \mathrm{KRT}^{+}$double-positive cells (figure 1c; dashed arrow; and figure S2B). We further detected increased amounts of $\gamma \mathrm{H} 2 \mathrm{~A} . \mathrm{X}$ (phospho S139), a marker for DNA double strand breaks and activated DNA damage response, which has been implicated in cellular senescence, in epithelial cells of IPF patients co-localising with P16 staining (online supplementary figure S3). In addition, an increase in P16, but not P21 expression was detectable on the mRNA level in primary human ATII cells isolated from IPF patients compared to non-IPF donors (figure 1e). This discrepancy between changes in the P21 protein and gene expression level might be due to differential post-transcriptional control of P21 protein expression [29, 30]. Collectively, these data suggest that senescence occurs in the lung epithelium in IPF.

\section{Senescence markers are upregulated in experimental lung fibrosis}

Next, we analysed cellular senescence in mice subjected to bleomycin (Bleo)-induced lung fibrosis ( $2 \mathrm{U} \cdot \mathrm{kg}^{-1}$ body weight, sacrificed at day 7, 14 or 21 after instillation). Both P16 and P21 were significantly upregulated on the gene expression level in fibrotic mouse lungs (figure 2a; mean \pm SD $\triangle \mathrm{Ct} P 16$ : day 14 PBS $-5.66 \pm 0.34$ versus Bleo $-4.33 \pm 0.21 ; P 21$ : day 14 PBS $-0.64 \pm 0.22$ versus Bleo $1.42 \pm 0.22 ; \mathrm{p}<0.001$ ). While $P 16$ was upregulated as early as day 14 post-Bleo instillation, $P 21$ was upregulated earlier at day 7 and decreased back to baseline by day 21. The different kinetics of $P 16$ and $P 21$ expression were also observed in a previously published microarray dataset (online supplementary figure S4A) [34], and probably represent the different kinetics of $P 16 / P 21$ induction upon DNA damage [35, 36]. P21 protein expression was significantly increased at day 7 as analysed using Western blotting (figure 2b). Unfortunately, due to the lack of reliable and specific mouse P16 antibodies, we could not analyse P16 on protein level [11]. Next, we assessed SA- $\beta$-galactosidase activity, a widely used surrogate marker for the detection of 


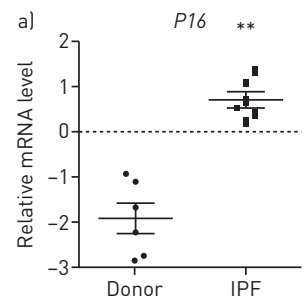

b)
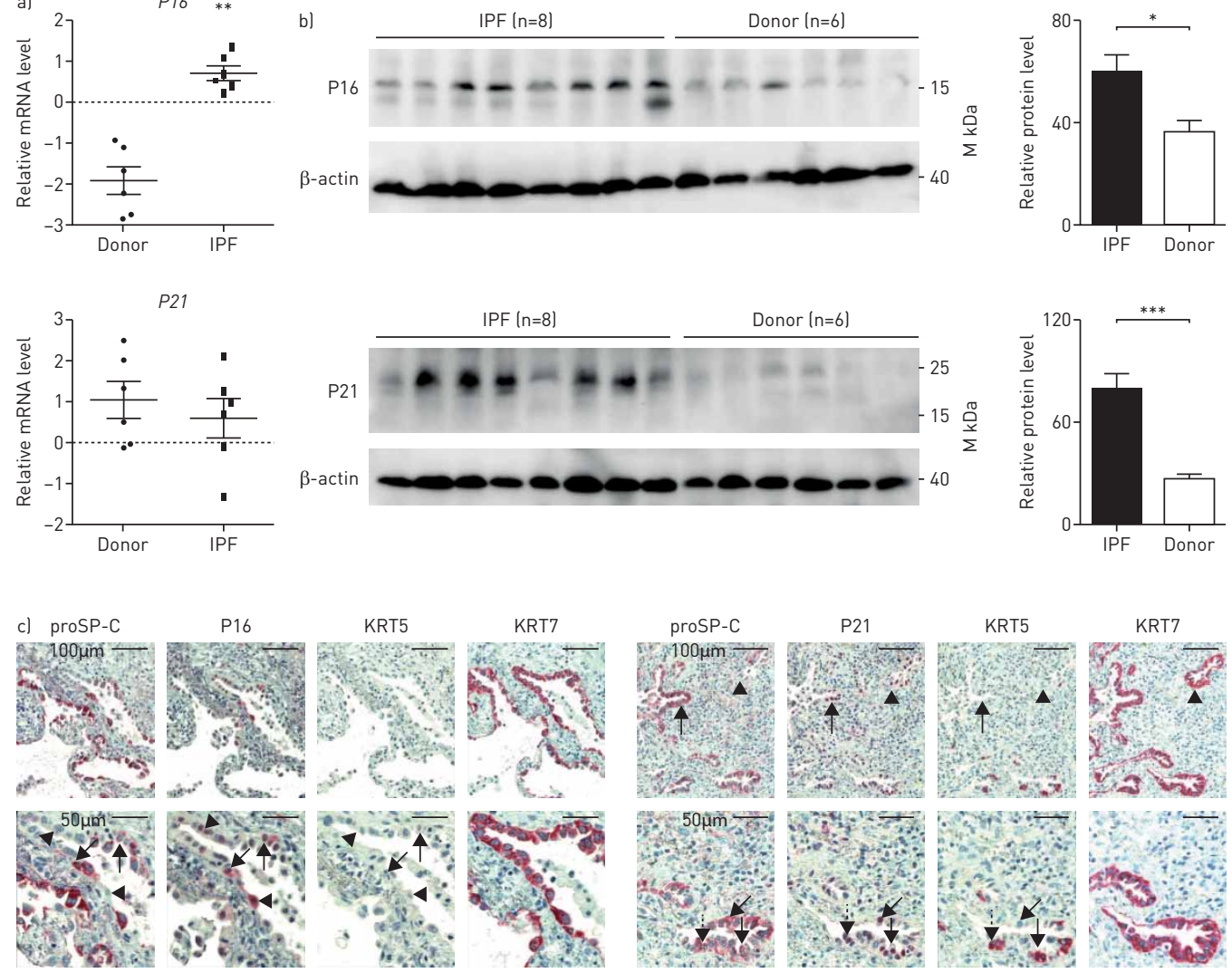

KRT5
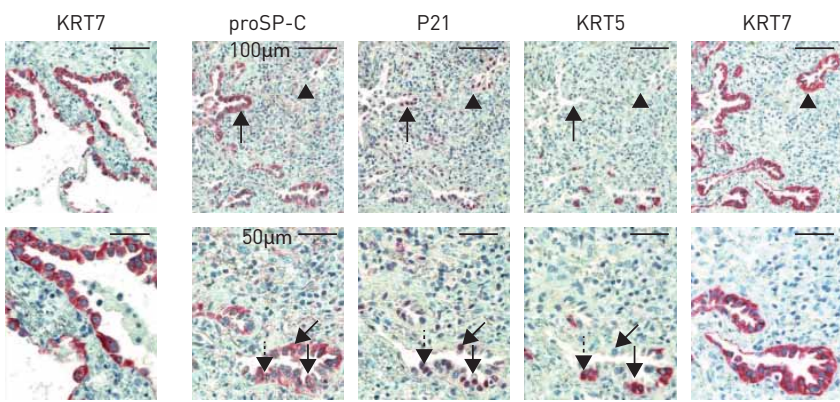

d) proSP-C

P16

KRT5

KRT7

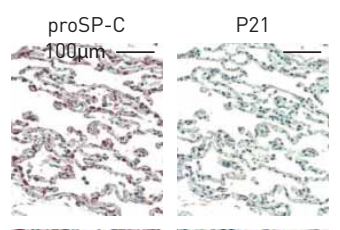

proSP-C
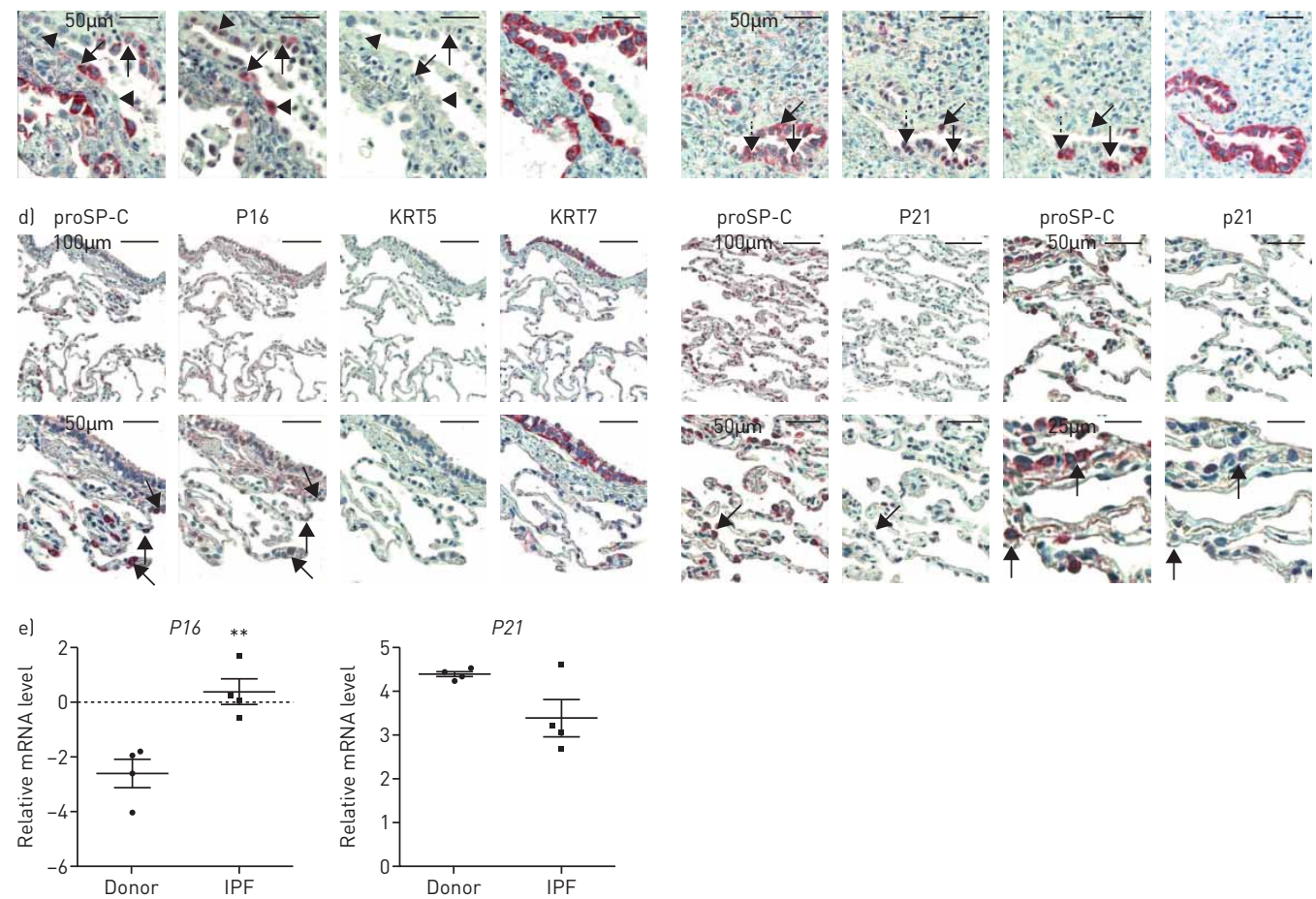

FIGURE 1 Senescence marker expression is upregulated in idiopathic pulmonary fibrosis (IPF) patients. a) Gene expression of $P 16$ and $P 21$ in lung homogenates of IPF and donor tissue was measured by quantitative (q)PCR and normalised to HPRT. Data are presented as mean \pm SEM. $n=6$. Means were compared using unpaired t-tests. b) Representative and quantitative immunoblot analyses of subpleural lung tissue from patients with sporadic IPF ( $n=16)$ and human donor lungs ( $n=11)$ using specific antibodies against P16 and P21, and $\beta$-actin as loading control. Densitometric ratios of the respective protein to $\beta$-actin are given as mean \pm SEM. Means were compared using unpaired t-tests. Immunohistochemical staining of serial sections of c) IPF or d) donor lung tissue for prosurfactant protein-C (proSP-C; marker for alveolar epithelial type (AT)II cells), cytokeratin 5 (KRT5, marker for bronchiolar basal cells), cytokeratin 7 (KRT7, marker for simple epithelial cells) and P16 and P21 protein. ProSP-C ${ }^{+} \mathrm{KRT7}^{+}$ATII cells expressing P16 or P21 are indicated by arrows; proSP-C ${ }^{-} \mathrm{KRT5}^{-}$ $\mathrm{KRT7}^{+}$epithelial cells expressing P16 or P21 are indicated by arrowheads; proSP-C ${ }^{+} \mathrm{KRT5}^{+} \mathrm{KRT7}^{+}$epithelial cells expressing P21 are indicated by dashed arrows. e) Gene expression of P16 and P21 in primary human ATIl cells isolated from IPF and donor tissue was measured using qPCR and normalised to HPRT. Data are presented as mean \pm SEM. $n=4$. Means were compared using unpaired t-tests. ${ }^{*}: p<0.05 ;{ }^{* *}: p<0.01 ;{ }^{* * *}: p<0.001$. 

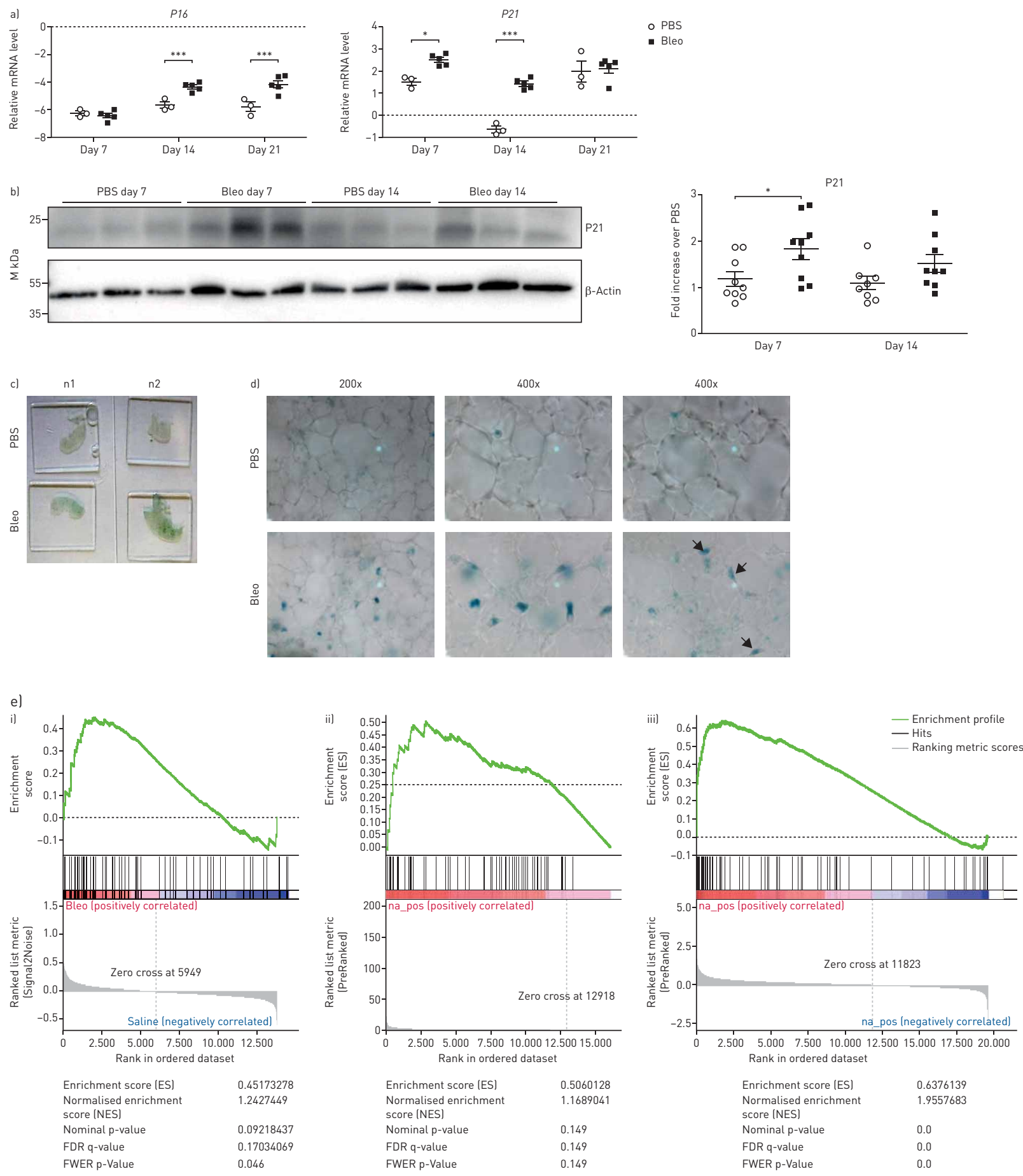

FIGURE 2 Senescence markers are upregulated in experimental lung fibrosis. Mice were instilled with PBS or bleomycin (Bleo) and sacrificed at the timepoints indicated. a) Gene expression of $P 16$ and $P 21$ in lung homogenates of mice sacrificed at day 7, 14 or 21 was measured using quantitative PCR and normalised to Hprt. Change in cycle threshold $(\Delta C t)$ is presented as mean \pm SEM; $n=3$ for PBS and $n=5$ for Bleo. Statistical significance was tested using one-way ANOVA followed by Newman-Keuls' multiple comparison test. b) Immunoblot of P21 protein in mouse whole-lung homogenates of mice treated with PBS or Bleo and sacrificed after 7 or 14 days. $\beta$-Actin was used as a loading control. Respective sizes of marker are indicated. Data were quantified and normalised to loading control. Data are presented as mean \pm SEM; $\mathrm{n}=9$. Statistical significance was tested using one-way ANOVA followed by Newman-Keuls' multiple comparison test. c, d) Three-dimensional lung tissue cultures (3D-LTCs) were obtained from mice instilled with PBS or Bleo and sacrificed at day 14. 3D-LTCs were stained for senescence-associated $\beta$-galactosidase activity and c) macroscopic images and d) microscopic (magnification of 200x or 400x) images were taken. Epithelial cells are marked by arrows. e) Enrichment of senescence-associated genes [31] in microarray data of i) whole lung [32] (GSE16846), ii) mouse fibroblasts [33] (GSE42564) or iii) primary mouse (pm) alveolar epithelial type (AT)II cells [26] of mice with experimental lung fibrosis induced by Bleo. FDR: false discovery rate; FWER: family-wise error rate. ${ }^{*}: p<0.05 ;{ }^{* * *}: p<0.001$. 
senescent cells [28], in three-dimensional lung tissue cultures (3D-LTCs) from fibrotic mouse lungs (day 14 post-Bleo). Importantly, increased blue staining, indicating a higher number of senescent cells, was observed in fibrotic lungs as compared to healthy lungs (figure $2 \mathrm{c}$ ). The SA- $\beta$-galactosidase activity was predominantly observed in structural cells that morphologically resembled lung epithelial cells (figure 2d; arrows). To further explore whether senescence is increased and in which cell types senescence occurs in experimental lung fibrosis, we utilised gene set enrichment analysis [37] of previously published microarray data sets obtained from whole murine lungs [32], primary murine fibroblasts ( $\mathrm{pmFb}$ [33] or pmATII cells [26] of Bleo- versus PBS-treated mice and compared those to a previously published gene signature list for senescence [31] (figure 2e). We found a significant enrichment of senescence-associated genes in fibrotic pmATII cells, but not in whole lung or pmFb from Bleo-treated mouse lungs (figure 2e). These data demonstrate that senescence-associated genes are enriched in experimental lung fibrosis and indicate that the lung epithelium is a potential source of senescent cells in the fibrotic lung.

\section{Increased senescence of ATII cells in experimental lung fibrosis}

To further analyse whether ATII cells are a major senescent cell type in lung fibrosis, we isolated pmATII cells from Bleo (fibrotic) and PBS (healthy) mouse lungs (figure 3a and online supplementary figure S4B). Notably, freshly isolated fibrotic EpCAM ${ }^{+}$pmATII cells exhibited increased staining for SA- $\beta$-galactosidase activity over control EpCAM ${ }^{+}$pmATII cells, as analysed using flow cytometry (figure $3 \mathrm{~b}$ ). After $48 \mathrm{~h}$ of culture, fibrotic ATII cells maintained an increase of $2.45 \pm 0.45$-fold in SA- $\beta$-galactosidase staining over healthy cells (figure $3 \mathrm{c}$ ). This was further confirmed by conventional light microscopy following in vitro culture and SA- $\beta$-galactosidase staining (figure $3 \mathrm{~d}$ ). Accordingly, freshly isolated fibrotic pmATII cells showed increased P16 and P21 transcript levels as well as senescence-associated heterochromatic foci marked by foci of histone $\mathrm{H} 3$ lysine 9 trimethylation (H3K9me3) (figure 3e and online supplementary figure S4C). Additionally, upregulation of secreted phosphoprotein (Spp) 1 and matrix metalloproteinase $(M m p)$ 2, two well-known components of the SASP, was observed (figure 3e). In order to examine whether P16 and P21 activation translated to increased SASP activity, we performed a proteomic analysis of the secretome of fibrotic and normal pmATII cells. We identified several SASP components in our screen and found that $52 \%$ of those identified were upregulated $>1.5$-fold in the fibrotic secretome, whereas only $10 \%$ of detected SASP components were downregulated (figure $3 \mathrm{f}$ and $\mathrm{g}$ ). Among the most upregulated SASP components were insulin growth factor binding proteins (Igfbp) 3, 4 and 7 and MMP 3, 12 and 14 (figure $3 \mathrm{~h}$ ). Together, these data strongly suggest increased senescence of fibrotic ATII cells along with increased secretion of SASP factors.

\section{Depletion of senescent cells by senolytic drugs decreases fibrotic markers and increases epithelial cell marker expression}

Whether senescence contributes to or limits pulmonary fibrosis is still an area of active discussion. To address this point, we used a recently described combination of senolytic drugs, dasatinib (D) and quercetin (Q) (a tyrosine kinase inhibitor and flavonol combination) to deplete senescent cells from culture [39-41]. Fibrotic pmATII cells exhibited stable expression of profibrotic markers during culture (online supplementary figure S5A). Treatment with senolytic compounds reduced total cell numbers and the percentage of senescent cells (figure $4 \mathrm{a}$ and b) and P16 expression level dropped significantly (figure 4c). Importantly, an increase in apoptotic cleaved caspase 3 and annexin V staining was observed in ATII cells upon senolytic treatment (figure $4 \mathrm{~d}$ and e). Apoptosis was predominantly induced in senescent cells (figure 4f), consistent with a depletion of senescent cells induced by senolytic drugs [41].

We next analysed whether senolytic treatment affected SASP factors in pmATII cells. Notably, treatment with DQ led to a reduction of SASP factors such as Mmp12, Serpine1 and Spp1 (figure 4g). Senescent cell depletion further correlated with reduced extracellular matrix components Collagen1a1, Collagen $5 a 3$ and Fibronectin (figure 5a and online supplementary figure S5C), which have been suggested to be part of the SASP [22, 35]. Notably, we found significantly increased mRNA expression of the epithelial cell marker E-cadherin (Cdh1) as well as functional ATII cell markers, such as Sftpc and Sftpa, while the ATI cell marker $T 1 \alpha$ was unaffected (figure $5 \mathrm{~b}$ and online supplementary figure S5D). In addition, protein secretion of SP-C was increased upon senolytic treatment (figure 5e) along with increased E-cadherin protein level (figure 5f). We further analysed interleukin (IL)-6 protein secretion (a major component of the SASP), and found significantly decreased amounts upon senolytic treatment (figure 5c; DMSO $3.95 \pm 0.81$-fold over control versus DQ $1.34 \pm 0.65$-fold over control). Moreover, transcript level as well as secretion of the ATII cell-derived fibrotic mediator Wnt-inducible signalling protein (Wisp) 1 [26] were significantly reduced upon senolytic treatment (online supplementary figure S5C and figure 5d; DMSO $2.10 \pm 0.91$-fold over control versus DQ $0.71 \pm 0.26$-fold over control). Together, these data suggest effective depletion of senescent cells and modulation of their associated SASP. Notably, when treating ATII cells isolated from PBS-treated lungs, which show reduced senescence as compared to fibrotic ATII cells, with 

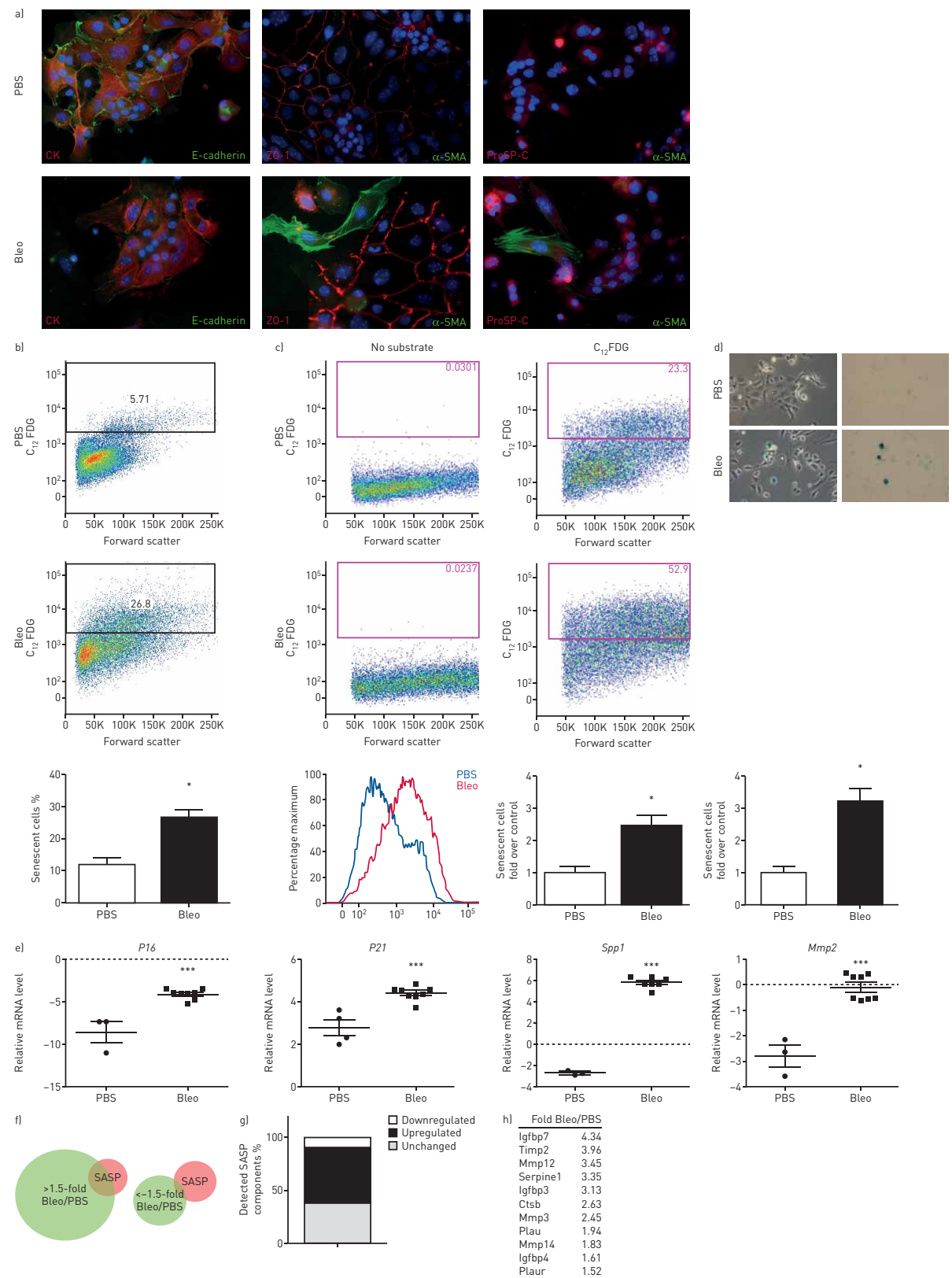

FIGURE 3 Senescence markers are upregulated in alveolar epithelial type (AT) II cells in experimental lung fibrosis. Mice were instilled with either PBS or bleomycin (Bleo). At day 14 after instillation, mice were sacrificed and primary mouse (pm)ATII cells were isolated. a) Immunofluorescence staining of fibrotic or nonfibrotic pmATIl cells on cover slips for epithelial cell marker expression at day 2 after isolation. Fluorescent images represent a 400x magnification. b) pmATII cells were analysed for epithelial cell adhesion molecule (EpCAM) positivity and senescence-associated (SA)- $\beta$-galactosidase activity by fluorescence-activated cell sorting (FACS) directly after isolation. Representative dot blots of the EpCAM ${ }^{+}$population are shown for PBS and Bleo, as well as quantifications of percentages of senescent cells of the EpCAM ${ }^{+}$population. Means were compared to time-matched PBS controls using unpaired t-tests; $n=3$. c) pmATIl cells (day 2) were analysed for SA- $\beta$-galactosidase activity using FACS. Representative dot blots are shown for PBS and Bleo pmATII cells incubated with $\mathrm{C}_{12}$ FDG or respective controls, a representative histogram comparing PBS and Bleo pmATII cells incubated with $\mathrm{C}_{12}$ FDG as well as quantifications of percentages of senescent cells normalised to respective PBS control. Means were compared to time-matched PBS controls using unpaired t-tests; $\mathrm{n}=3$. d) pmATII cells (day 2) were stained for SA- $\beta$-galactosidase activity and blue cells and total cells were counted. Representative images and quantitative data normalised to respective PBS controls are shown. Data represent mean士SEM. Means were compared to time-matched PBS controls using unpaired t-tests; $n=3$. e) Gene expression of senescence-associated genes in freshly isolated pmATIl cells from PBS- or Bleo-treated mice was measured using quantitative PCR. Data were normalised to Hprt. Change in threshold cycle ( $\Delta \mathrm{C} t)$ is presented as mean \pm SEM; $n=3-4$ for PBS and $\mathrm{n}=8$ for Bleo. Means were compared to time-matched PBS controls using unpaired t-tests. $\mathrm{f}-\mathrm{h}$ ) pmATIl cells isolated from PBS- or Bleo-treated mice were plated onto plastic tissue culture plates. After $48 \mathrm{~h}$ of culture, the supernatant was collected and analysed using mass spectrometry proteomics. The senescence-associated secretory phenotype (SASP) list [9] was compared to the list of secreted proteins (1.5-fold upregulated or downregulated). f) A Venn diagram (BioVenn; [38]) showing the overlap of SASP proteins with the up-/downregulated proteins in the pmATII supernatant. g) Percentage of detected SASP factors that are upregulated ( $>1.5$-fold) or downregulated $(<-1.5$-fold) or not changed. h) List of upregulated SASP components $>1.5$-fold Bleo/PBS. CK: cytokeratin; ZO: zona occludens; SMA: smooth muscle actin. ${ }^{*}: p<0.05$; ${ }^{* * *}: p<0.001$. 

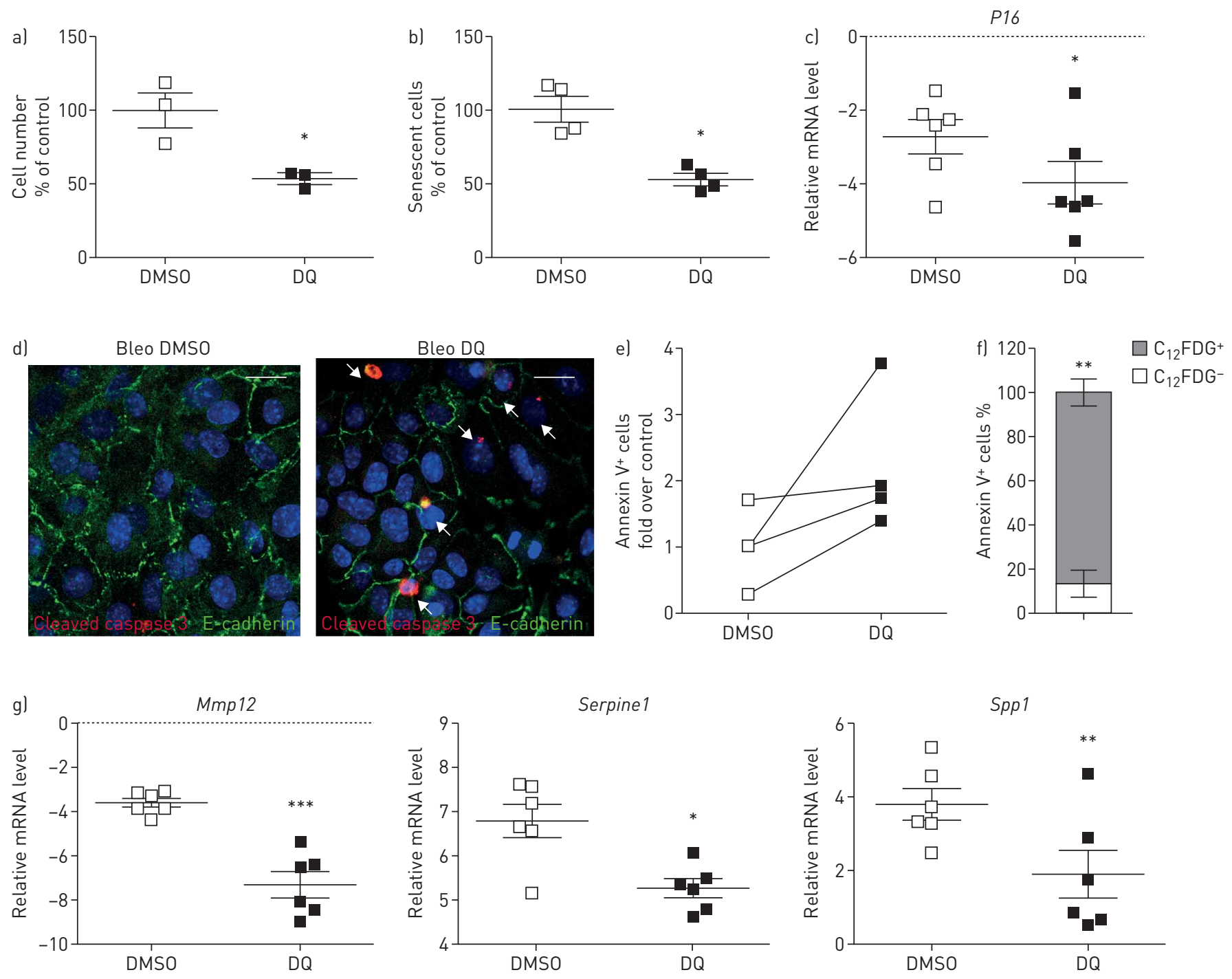

FIGURE 4 Treatment of fibrotic primary mouse (pm) alveolar epithelial type (AT)II cells with senolytic drugs decreases senescent markers and increases apoptosis. Mice were instilled with either PBS or bleomycin (Bleo). At day 14 after instillation mice were sacrificed and pmATII cells were isolated. Fibrotic pmATII cells were cultured for $48 \mathrm{~h}$ in the presence of the senolytic drugs dasatinib (D; $200 \mathrm{nM})$ and quercetin $(Q ; 50 \mu M)$. a) The senolytic activity was assessed by cell numbers. Data are presented as normalised to dimethylsulfoxide (DMSO) control and as mean \pm SEM. Significance was assessed using paired t-tests; $n=3$. b) Senescence-associated (SA)- $\beta$-galactosidase activity. pmATII cells were stained for SA- $\beta$-galactosidase activity and blue cells and total cells were counted. Quantitative data are normalised to respective DMSO control. Data are presented as mean \pm SEM. Means were compared to time-matched controls using paired t-tests; $n=4$. c) Gene expression analysis for the senescence marker P16. Data were normalised to Hprt level. Data are presented as normalised to DMSO control and as mean \pm SEM. Significance was assessed with paired t-tests; $n=6$. d) Representative images of immunofluorescence staining for apoptotic marker cleaved caspase 3 and E-cadherin in fibrotic pmATII cells exposed to DMSO or DQ. Fluorescent images represent a $630 \times$ magnification. Scale bars $=20 \mu m$. e) Fibrotic ATII cells were exposed to DMSO or DQ and stained for annexin $\mathrm{V}$ level and analysed using fluorescence-activated cell sorting (FACS); $\mathrm{n}=4$. f) Fibrotic ATII cells were exposed to $D Q$ and stained for senescence $\left(\mathrm{C}_{12} \mathrm{FDG}\right)$, co-stained for annexin $V$ level and analysed using FACS. Data are presented as mean \pm SEM percentage of total apoptotic cells in the senescent $\left(\mathrm{C}_{12} \mathrm{FDG}^{+}\right)$and nonsenescent $\left(\mathrm{C}_{12} \mathrm{FDG}^{-}\right)$population. Significance was assessed using unpaired t-tests; $n=3$. g) Expression of senescence-associated secretory phenotype (SASP) markers in pmATIl cells treated with senolytic drugs was analysed using quantitative PCR. Data were normalised to Hprt level. Change in threshold cycle $(\Delta \mathrm{Ct})$ is presented as mean \pm SEM. Significance was assessed using paired t-tests; $n=6$. Spp: secreted phosphoprotein; Mmp: matrix metalloproteinase. ${ }^{*}: p<0.05 ;{ }^{* *}: p<0.01$; $* * *: p<0.001$.

senolytic drugs, we only observed a slight reduction in cell number, P16 expression and SASP components accompanied by an increase in epithelial cell markers as compared to control (online supplementary figure S6).

Finally, we aimed to elucidate whether depletion of senescent cells further modulates fibrotic burden in an ex vivo model using native lung tissue slice cultures. 3D-LTCs derived from Bleo-treated mouse lungs exhibit increased expression of fibrotic marker (online supplementary figure S7A), as well as senescence-associated P16 and P21 expression (figure 6a). Treatment of fibrotic 3D-LTCs with senolytic 

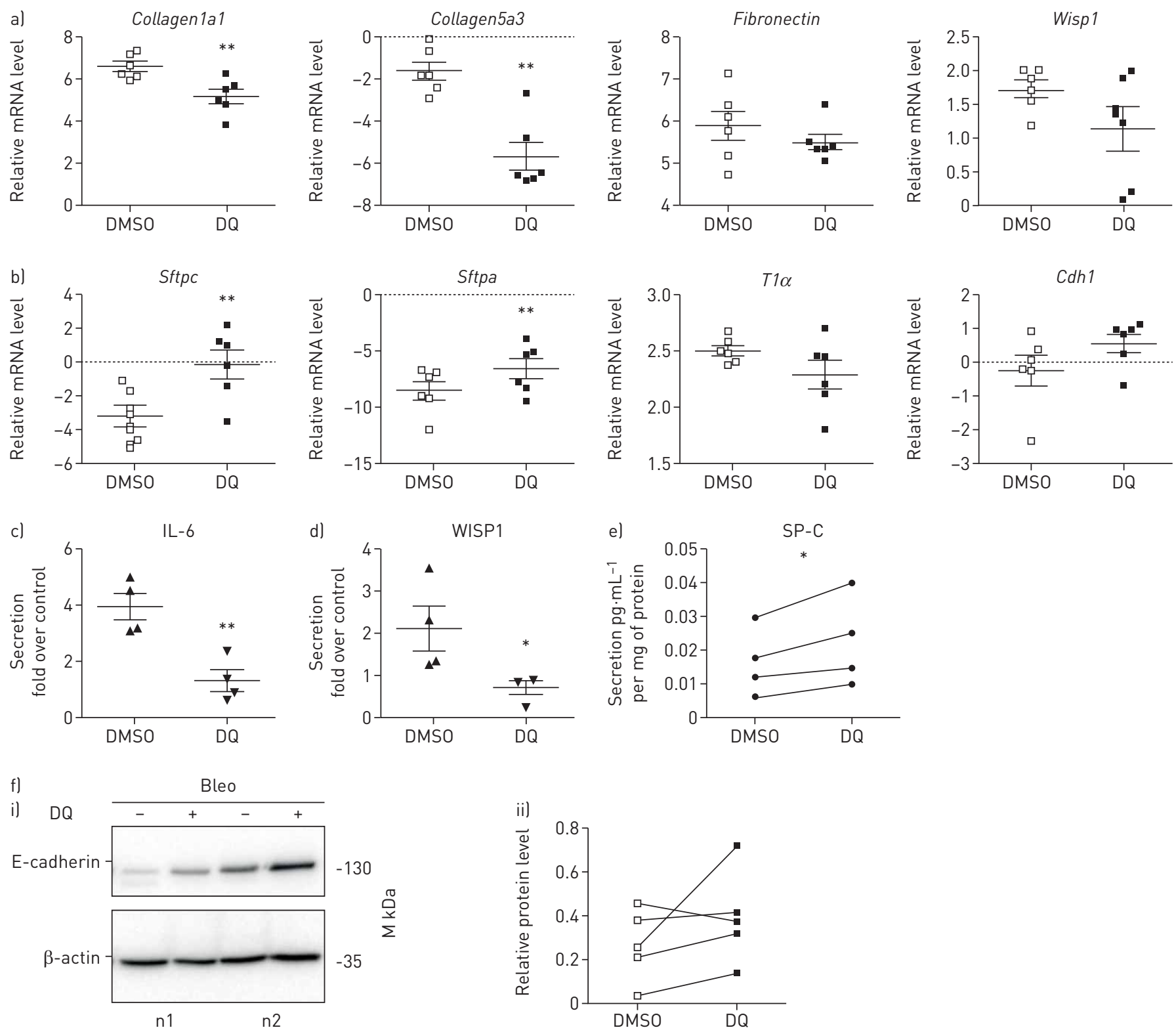

FIGURE 5 Treatment of fibrotic primary mouse (pm) alveolar epithelial type (AT)II cells with senolytic drugs decreases fibrotic and increases epithelial cell markers. Mice were instilled with either PBS or bleomycin (Bleo). At day 14 after instillation, mice were sacrificed and pmATII cells were isolated. Fibrotic pmATII cells were cultured for $48 \mathrm{~h}$ in the presence of senolytic drugs dasatinib (D; $200 \mathrm{nM})$ and quercetin $(Q ; 50 \mu M)$. a) Expression of fibrotic markers was analysed using quantitative (q)PCR. Data were normalised to Hprt level. Change in threshold cycle ( $\Delta$ Ct) is presented as mean \pm SEM. Significance was assessed using paired t-tests; $n=6$. b) Expression of epithelial markers was analysed by qPCR. Data were normalised to Hprt level. $\Delta \mathrm{C}$ t is presented as mean \pm SEM. Significance was assessed using paired $\mathrm{t}$-tests; $\mathrm{n}=6$. c) Secretion of interleukin (IL)-6 was analysed using ELISA. Data are presented as normalised to PBS control treated with dimethylsulfoxide (DMSO) (mean \pm SEM). Significance was assessed using paired t-tests; $n=4$. d) Secretion of Wnt-inducible signalling protein (WISP) 1 in pmATII cells treated with senolytic drugs was analysed using ELISA. Data are presented as normalised to PBS control treated with DMSO (mean士SEM). Significance was assessed using t-tests; $n=4$. e) Secretion of surfactant protein-C (SP-C) was analysed using ELISA. Data are presented as normalised to total cell protein amount. Significance was assessed using paired t-tests; $n=4$. f) i) E-cadherin expression was assessed using Western blotting. $\beta$-actin was used as a loading control; ii) quantification of E-cadherin Western blot; $n=5$. Data were normalised to $\beta$-actin. ${ }^{*}: p<0.05 ;{ }^{* *}: p<0.01 ;{ }^{* *}: p<0.001$.

drugs reduced SA- $\beta$-galactosidase staining and P16 expression (figure $6 \mathrm{~b}$ and $\mathrm{c}$ ) and increased cleaved caspase 3 staining (figure $6 \mathrm{~d}$ ). In addition, senolytic treatment reduced expression of the SASP components Mmp12, Serpine1 and Spp1 (figure 6e). Importantly, DQ treatment further reduced fibrotic markers, such as Collagenla1 and Wisp1 transcript and protein levels (figure 7a, $\mathrm{c}$ and e), whereas Sftpc transcript and protein expression increased compared to time-matched control (figure $7 \mathrm{~b}$ and d). Notably, we observed similar trends when treating healthy 3D-LTCs with senolytic drugs, albeit to a lower extent than in the fibrotic 3D-LTCs (online supplementary figure S8). In summary, senescent cell depletion by 


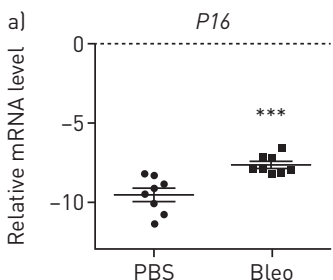

b)

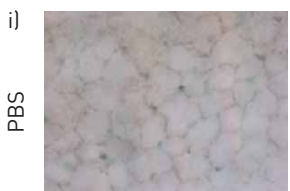

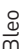

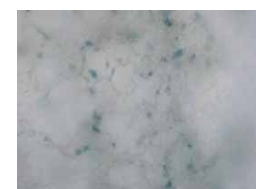

음

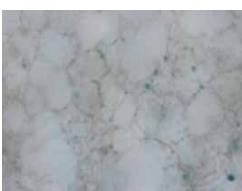

$\frac{\odot}{\infty}$

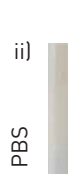

D

$\mathrm{DQ}$

$\stackrel{\infty}{\alpha}$
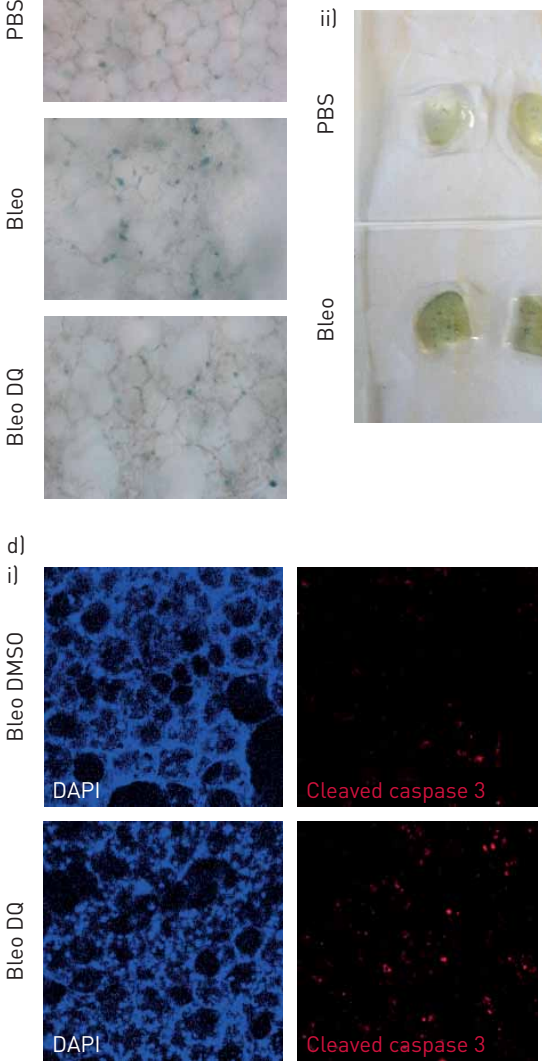
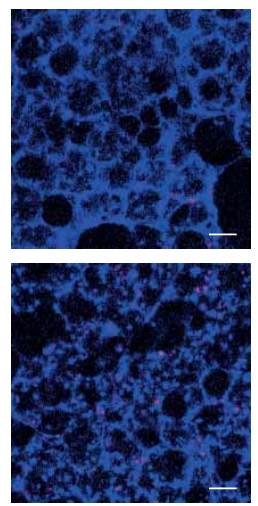

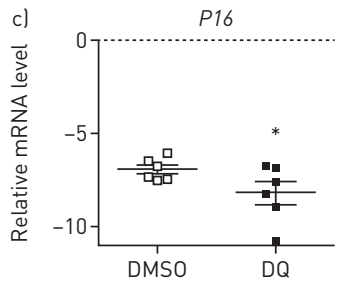

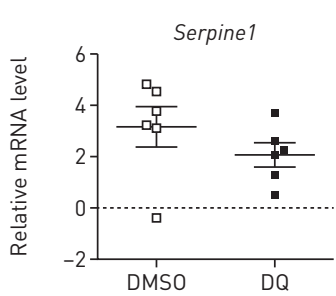

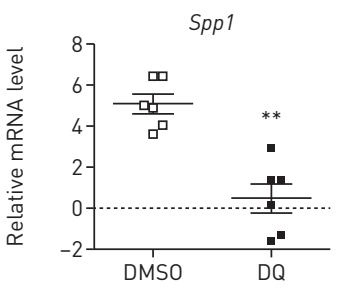

FIGURE 6 Treatment of fibrotic three-dimensional lung tissue cultures (3D-LTCS) with senolytic drugs decreases senescence markers and increases apoptosis markers. Mice were instilled with either PBS or bleomycin (Bleo). At day 14 after instillation mice were sacrificed and 3D-LTCs were generated. al Gene expression of senescence markers in 3D-LTCs after $48 \mathrm{~h}$ of culture was analysed using quantitative (q)PCR. Data were normalised to Hprt. Change in threshold cycle $(\Delta \mathrm{Ct})$ is presented as mean \pm SEM. Significance was assessed using unpaired t-tests; $n=8$. b-e) Fibrotic 3D-LTCs were cultured for $48 \mathrm{~h}$ in the presence of senolytic drugs dasatinib (D;200 nM) and quercetin $(Q ; 50 \mu \mathrm{M})$. The senolytic activity was assessed using b) senescence-associated $\beta$-galactosidase staining (i) 200x), as well as by c) gene expression analysis for the senescence marker P16. Data were normalised to Hprt. $\Delta \mathrm{Ct}$ is presented as mean \pm SEM. Significance was assessed using paired t-tests; $n=6$. d) Representative images of immunofluorescence staining for the apoptotic marker cleaved caspase 3. i) Scale bars $=50 \mu \mathrm{m}$; ii) scale bars $=20 \mu \mathrm{m}$. Epithelial cells are marked by arrows. e) Gene expression of SASP markers in 3D-LTCs treated with senolytic drugs was analysed using qPCR. Data were normalised to Hprt. $\triangle \mathrm{Ct}$ is presented as mean \pm SEM. Significance was assessed using paired t-tests; $n=6 .{ }^{*}: p<0.05 ; * *: p<0.01{ }^{* * *}: p<0.001$. 

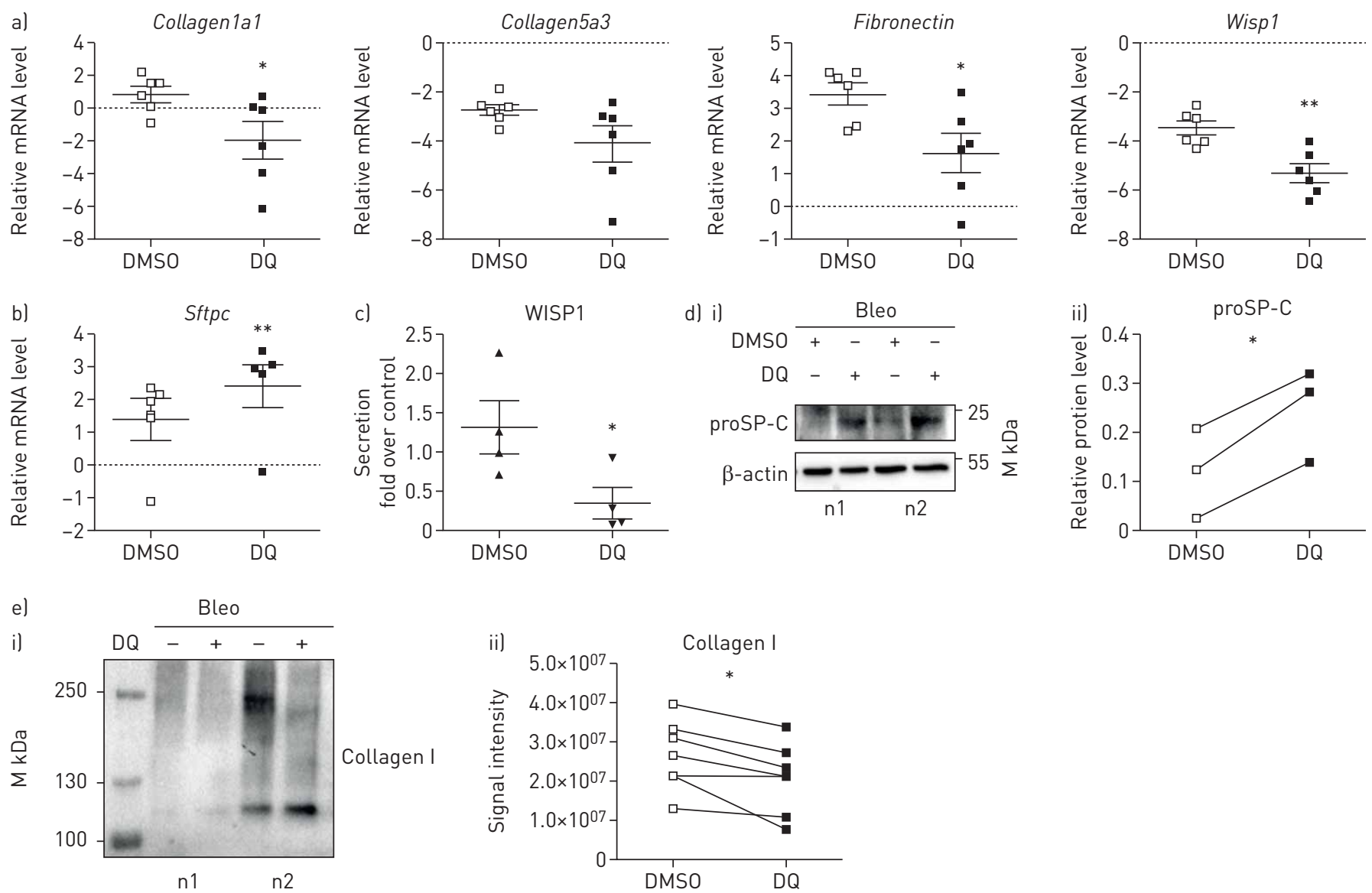

FIGURE 7 Treatment of fibrotic three-dimensional lung tissue cultures (3D-LTCs) with senolytic drugs decreases fibrotic and increases epithelial cell markers. Mice were instilled with either PBS or bleomycin (Bleo). At day 14 after instillation mice were sacrificed and 3D-LTCs were generated. Fibrotic 3D-LTCs were cultured for $48 \mathrm{~h}$ in the presence of senolytic drugs dasatinib (D; $200 \mathrm{nM}$ ) and quercetin (Q; $50 \mu \mathrm{M}$ ). a) Gene expression of fibrotic markers in 3D-LTCs treated with senolytic drugs was analysed using quantitative (q)PCR. Data were normalised to Hprt. Change in threshold cycle $(\Delta \mathrm{Ct})$ is presented as mean \pm SEM. $n=6$. b) Gene expression of Sftpc in 3D-LTCs treated with senolytic drugs was analysed using qPCR. Data were normalised to Hprt. $\Delta$ Ct is presented as mean \pm SEM. $n=5$. c) Secretion of Wnt-inducible signalling protein (WISP)1 from 3D-LTCs treated with senolytic drugs was analysed using ELISA. Data are presented as normalised to PBS dimethylsulfoxide (DMSO) control (mean \pm SEM). $n=4$. d) i) Prosurfactant protein-C (proSP-C) expression was assessed using Western blotting in fibrotic 3D-LTCs. $\beta$-actin was used as a loading control; ii) quantification of proSP-C protein relative to $\beta$-actin. $n=3$. e) i) Secreted collagen I was assessed using Western blotting in fibrotic 3D-LTCs; ii) quantification of secreted collagen I normalised to supernatant volume. $n=7$. Significance was assessed using paired t-tests. $*: p<0.05 ;{ }^{* *}: p<0.01 ;{ }^{* * *}: p<0.001$.

senolytic drugs reduced fibrotic burden and increased ATII cell markers in primary ATII cells as well as in ex vivo 3D-LTCs.

\section{Discussion}

IPF is a disease of the elderly, and several hallmarks of ageing such as cellular senescence have been linked to this disease [5]. Recently, BAKER et al. [42] were able to demonstrate that depletion of naturally occurring senescent cells extends healthy lifespan and decreases age-induced pathologies in mice. Nevertheless, there is evidence that senescence might also limit diseases, such as cancer or fibrotic disorders of the skin or heart [10-12]. Recent reports in lung fibrosis are conflicting, since both a detrimental role [17, 21, 22, 43] as well as an antifibrotic role [23] have been reported. In this study, we utilised senolytic drugs on fibrotic lung epithelial cells in vitro and ex vivo in 3D-LTCs and demonstrated that senolytic treatment attenuates fibrotic mediator expression, while stabilising epithelial cell marker expression and function. These findings suggest that senescence contributes to development of lung fibrosis and that treatment of pulmonary fibrosis with senolytic drugs might be beneficial.

Increased senescence has been described for IPF as well as in mouse models of pulmonary fibrosis in both epithelial cells and (myo)fibroblasts $[16-18,21,23,44-46]$. Here, we confirm that lung epithelial cells from experimental and human IPF exhibit increased cellular senescence. Interestingly, we observed cellular senescence in different subpopulations of human lung epithelial cells, including a population of proSP-C $\mathrm{C}^{+}$ $\mathrm{KRT5}^{+}$double-positive cells. These double-positive cells have been described in the mouse as derived from 
a rare undifferentiated epithelial cell population, which is activated upon influenza infection or bleomycin challenge [47]; however, the origin of these cells as well as the contribution of single cell subpopulations in the human lung to disease pathogenesis requires further investigation.

Senescent cells secrete several mediators in the SASP that have been shown to directly influence their surrounding microenvironment. Importantly, we identified fibrotic ATII cells as a potent source of profibrotic SASP components. In line with this, increased expression of SASP components has been found in bleomycin-induced lung fibrosis in vivo $[22,44]$. Different components of the SASP such as IL-6, MMP-12 [48], IL-1 $\beta$ [49] or keratinocyte growth factor [50] have been described to induce alveolar epithelial cell reprogramming, a prominent feature of IPF pathogenesis $[2,51]$. Notably, several different epithelial cell phenotypes can be observed in the fibrotic lung, including cellular senescence [51]. The distinct phenotypes are most likely determined by 1 ) cell intrinsic properties that differ in specific (and to be characterised) subpopulations and 2) extrinsic factors, such as the direct microenvironment. Components of the SASP have been described to influence cell proliferation [13]. Thus, it may be that the SASP of senescent fibrotic cells contributes to the hyperproliferative phenotype of some epithelial cells or to neighbouring fibroblasts. By depleting senescent epithelial cells, we were able to reduce their associated SASP, which had potent antifibrotic effects and partly restored the normal epithelial cell phenotype. While our results strongly support the hypothesis that senescent epithelial cells and their SASP contribute to fibrosis pathogenesis, it will be important to further elucidate specific SASP compositions of different cell types which might account for distinct outcomes in fibrotic diseases in further studies.

Different mechanisms can lead to the induction of senescence [10]. DNA damage, as well as telomere shortening, can trigger senescence. Notably, telomere attrition is a driving force in IPF and mutations in telomerase genes have been found in familial and sporadic cases of IPF [52, 53]. NAIKAWADi et al. [24] reported that telomere dysfunction in ATII cells, but not mesenchymal cells, led to increased cellular senescence and lung fibrosis. The same group previously found that alveolar epithelial cell senescence is regulated by microRNA-34a [16], which has also been reported to regulate senescence of lung fibroblasts [23]. In addition to DNA damage- or telomere shortening-induced senescence, overexpression of the canonical WNT mediator $\beta$-catenin can result in oncogene-induced senescence and WNT signalling has further been demonstrated in senescence occurring during embryonic development [54, 55]. Several WNT ligands, such as WNT3A, have been shown to induce senescence upon prolonged cellular exposure [56]. In line with this, increased WNT/ $\beta$-catenin activity has been reported in human and experimental lung fibrosis [26, 56-59]. Here we found that the expression of the WNT target WISP1 was reduced by senolytic treatment of fibrotic ATII cells, thus suggesting that WISP1 might contribute to the profibrotic SASP.

Depletion of senescent cells presents a potential therapeutic option for the treatment of several chronic diseases, including those of the ageing lung. Pharmacological targeting of senescent cells has been recently developed $[39,41]$. Both pharmacological approaches target antiapoptotic pathways that senescent cells are highly reliant on. Inhibiting these antiapoptotic pathways induces apoptosis in the senescent cells, however, while the drugs target predominantly senescent cells, other mechanisms cannot be excluded $[39,41,60]$. Here, we provide evidence that senolytic treatment induces apoptosis in senescent alveolar epithelial cells, which subsequently led to an attenuation of profibrotic marker expression and increased epithelial cell function. Concerns that antisenescent therapies might increase the risk of cancer have been addressed by a study showing that depletion of senescent cells actually reduced tumour burden in naturally aged animals, rather than increasing it [42].

Why senescent cells exhibit antifibrotic properties in some fibrotic disorders, while appearing to be detrimental in pulmonary fibrosis is an intriguing question. One explanation might be that distinct cell types are affected by senescence [10, 20,35]. In the case of liver, heart and skin fibrosis, where senescence is thought to be beneficial, myofibroblasts are the major senescent cell type [10-12, 20,61]. However, in IPF we and others demonstrate that epithelial cells represent a major cell type that is affected by senescence $[16,18]$ and while we did not find fibroblasts to be affected to a large extent, other recent publications report senescence in fibroblasts in IPF as well [17, 22]. Interestingly, senescence induced by microRNA-34a in epithelial cells seems to promote fibrosis in aged animals, while it reduces fibrotic burden when lung fibroblast senescence is induced [23,43], indicating that epithelial cell senescence is indeed detrimental, whereas fibroblast senescence is protective. A recent study employed a combination of senolytic drugs in experimental lung fibrosis in vivo and report a reduction of senescent cells as well as fibrosis development [22], similar to our findings. However, their work focused primarily on examining the contribution of senescent fibroblasts using in vitro approaches and they did not examine epithelial cell behaviour in their model. We demonstrated that both senolytic drugs reduce the senescent cell burden and attenuated fibrotic marker using an ex vivo model of lung fibrosis in 3D-LTCs. This technique allows the analysis of tissue-level responses to senolytic drugs in living tissue ex vivo. Moreover, murine 3D-LTCs can 
be applied to extend mechanistic studies, while reducing overall animal experimentation. Notably, we were able to confirm our in vitro findings with respect to epithelial cell marker expression as well as fibrosis markers and provide evidence that epithelial cells are also targeted in 3D-LTCs. However, it is most likely that also other cell types, such as fibroblasts [22] are affected by senolytic treatment in this system. Future studies using in vivo models targeting specific senescent cell populations are needed to further delineate senescent cell-specific contribution to the development of pulmonary fibrosis. In addition, it will be important to further confirm the role of senescent cell types in human lung tissue. To this end, we have recently developed a model that induces early fibrotic-like changes in human 3D-LTCs from non-IPF patients [62], which may help us define the potential for as well as the limitations of antisenescent therapy in the context of lung fibrosis.

\section{Acknowledgements}

The authors are grateful to all members of the Königshoff Laboratory (Munich, Germany) for discussions and to Nadine Adam, Anastasia van den Berg, Julia Kipp, Maria Magdalena Stein and Rabea Imker for excellent technical assistance. Human samples were kindly provided by the Comprehensive Pneumonology Center Munich (CPC-M) BioArchive. This work would not have been possible without the contribution of the Biobank teams of Asklepios Klinikum (Munich-Gauting, Germany) and CPC, especially Ina Koch, Britta Peschel and Anja Stowasser.

Author contributions were as follows. M. Lehmann, K. Mutze, D.E. Wagner and M. Königshoff conceived and designed the research; M. Lehmann, K. Mutze, M. Korfei, S. Klee, D.E. Wagner, W. Skronska-Wasek, H.N. Alsafadi, C. Ota, R. Costa and H.B. Schiller planned and performed experiments and analysed the data; M. Korfei performed immunohistochemistry; H.B. Schiller performed mass spectrometry; A. Guenther and M. Lindner contributed tissue specimens and clinical expertise; M. Lehmann and M. Königshoff wrote the manuscript; K. Mutze, M. Korfei, D.E. Wagner and C. Ota edited the manuscript; and all authors approved the final version of the manuscript.

\section{References}

$1 \quad$ King TE Jr, Pardo A, Selman M. Idiopathic pulmonary fibrosis. Lancet 2011; 378: 1949-1961.

2 Wolters PJ, Collard HR, Jones KD. Pathogenesis of idiopathic pulmonary fibrosis. Annu Rev Pathol 2014; 9: 157-179.

3 King TE Jr, Bradford WZ, Castro-Bernardini S, et al. A phase 3 trial of pirfenidone in patients with idiopathic pulmonary fibrosis. N Engl J Med 2014; 370: 2083-2092.

4 Richeldi L, du Bois RM, Raghu G, et al. Efficacy and safety of nintedanib in idiopathic pulmonary fibrosis. $N$ Engl J Med 2014; 370: 2071-2082.

5 Meiners S, Eickelberg O, Königshoff M. Hallmarks of the ageing lung. Eur Respir J 2015; 45: 807-827.

6 Sueblinvong V, Neujahr DC, Mills ST, et al. Predisposition for disrepair in the aged lung. Am J Med Sci 2012; 344: 41-51.

7 Xu J, Gonzalez ET, Iyer SS, et al. Use of senescence-accelerated mouse model in bleomycin-induced lung injury suggests that bone marrow-derived cells can alter the outcome of lung injury in aged mice. J Gerontol A Biol Sci Med Sci 2009; 64: 731-739.

$8 \quad$ López-Otín C, Blasco MA, Partridge L, et al. The hallmarks of aging. Cell 2013; 153: 1194-1217.

9 Coppé JP, Desprez PY, Krtolica A, et al. The senescence-associated secretory phenotype: the dark side of tumor suppression. Annu Rev Pathol 2010; 5: 99-118.

10 Muñoz-Espín D, Serrano M. Cellular senescence: from physiology to pathology. Nat Rev Mol Cell Biol 2014; 15: 482-496.

11 Demaria M, Ohtani N, Youssef SA, et al. An essential role for senescent cells in optimal wound healing through secretion of PDGF-AA. Dev Cell 2014; 31: 722-733.

12 Krizhanovsky V, Yon M, Dickins RA, et al. Senescence of activated stellate cells limits liver fibrosis. Cell 2008; 134: 657-667.

13 Wang C, Jurk D, Maddick M, et al. DNA damage response and cellular senescence in tissues of aging mice. Aging Cell 2009; 8: 311-323.

14 Faner R, Rojas M, Macnee W, et al. Abnormal lung aging in chronic obstructive pulmonary disease and idiopathic pulmonary fibrosis. Am J Respir Crit Care Med 2012; 186: 306-313.

15 Tsuji T, Aoshiba K, Nagai A. Alveolar cell senescence in patients with pulmonary emphysema. Am J Respir Crit Care Med 2006; 174: 886-893.

16 Disayabutr S, Kim EK, Cha SI, et al. miR-34 miRNAs regulate cellular senescence in type II alveolar epithelial cells of patients with idiopathic pulmonary fibrosis. PLoS One 2016; 11: e0158367.

17 Hecker L, Logsdon NJ, Kurundkar D, et al. Reversal of persistent fibrosis in aging by targeting Nox4-Nrf2 redox imbalance. Sci Transl Med 2014; 6: 231 ra247.

18 Minagawa S, Araya J, Numata T, et al. Accelerated epithelial cell senescence in IPF and the inhibitory role of SIRT6 in TGF- $\beta$-induced senescence of human bronchial epithelial cells. Am J Physiol Lung Cell Mol Physiol 2011; 300: L391-L401.

19 Kuwano K, Kunitake R, Kawasaki M, et al. P21Waf1/Cip1/Sdi1 and p53 expression in association with DNA strand breaks in idiopathic pulmonary fibrosis. Am J Respir Crit Care Med 1996; 154: 477-483.

20 Selman M, López-Otín C, Pardo A. Age-driven developmental drift in the pathogenesis of idiopathic pulmonary fibrosis. Eur Respir J 2016; 48: 538-552.

21 Shivshankar P, Brampton C, Miyasato S, et al. Caveolin-1 deficiency protects from pulmonary fibrosis by modulating epithelial cell senescence in mice. Am J Respir Cell Mol Biol 2012; 47: 28-36.

22 Schafer MJ, White TA, Iijima K, et al. Cellular senescence mediates fibrotic pulmonary disease. Nat Commun 2017; 8: 14532

23 Cui H, Ge J, Xie N, et al. miR-34a inhibits lung fibrosis by inducing lung fibroblast senescence. Am J Respir Cell Mol Biol 2017; 56: 168-178. 
24 Naikawadi RP, Disayabutr S, Mallavia B, et al. Telomere dysfunction in alveolar epithelial cells causes lung remodeling and fibrosis. JCI Insight 2016; 1: e86704.

25 Uhl FE, Vierkotten S, Wagner DE, et al. Preclinical validation and imaging of Wnt-induced repair in human 3D lung tissue cultures. Eur Respir J 2015; 46: 1150-1166.

26 Königshoff M, Kramer M, Balsara N, et al. WNT1-inducible signaling protein-1 mediates pulmonary fibrosis in mice and is upregulated in humans with idiopathic pulmonary fibrosis. J Clin Invest 2009; 119: 772-787.

27 Yang J, Wheeler SE, Velikoff M, et al. Activated alveolar epithelial cells initiate fibrosis through secretion of mesenchymal proteins. Am J Pathol 2013; 183: 1559-1570.

28 Debacq-Chainiaux F, Erusalimsky JD, Campisi J, et al. Protocols to detect senescence-associated beta-galactosidase (SA-ßgal) activity, a biomarker of senescent cells in culture and in vivo. Nat Protoc 2009; 4: 1798-1806.

29 Abbas T, Dutta A. p21 in cancer: intricate networks and multiple activities. Nat Rev Cancer 2009; 9: 400-414.

30 Gong J, Ammanamanchi S, Ko TC, et al. Transforming growth factor $\beta 1$ increases the stability of p21/WAF1/CIP1 protein and inhibits CDK2 kinase activity in human colon carcinoma FET cells. Cancer Res 2003; 63: 3340-3346.

31 Fridman AL, Tainsky MA. Critical pathways in cellular senescence and immortalization revealed by gene expression profiling. Oncogene 2008; 27: 5975-5987.

32 Scotton CJ, Krupiczojc MA, Königshoff M, et al. Increased local expression of coagulation factor X contributes to the fibrotic response in human and murine lung injury. J Clin Invest 2009; 119: 2550-2563.

33 Tsukui T, Ueha S, Abe J, et al. Qualitative rather than quantitative changes are hallmarks of fibroblasts in bleomycin-induced pulmonary fibrosis. Am J Pathol 2013; 183: 758-773.

34 Peng R, Sridhar S, Tyagi G, et al. Bleomycin induces molecular changes directly relevant to idiopathic pulmonary fibrosis: a model for "active" disease. PLoS One 2013; 8: e59348.

35 Campisi J, d'Adda di Fagagna F. Cellular senescence: when bad things happen to good cells. Nat Rev Mol Cell Biol 2007; 8: 729-740.

36 Robles SJ, Adami GR. Agents that cause DNA double strand breaks lead to p16INK4a enrichment and the premature senescence of normal fibroblasts. Oncogene 1998; 16: 1113-1123.

37 Subramanian A, Tamayo P, Mootha VK, et al. Gene set enrichment analysis: a knowledge-based approach for interpreting genome-wide expression profiles. Proc Natl Acad Sci USA 2005; 102: 15545-15550.

38 Hulsen T, de Vlieg J, Alkema W. BioVenn - a web application for the comparison and visualization of biological lists using area-proportional Venn diagrams. BMC Genomics 2008; 9: 488.

39 Chang J, Wang Y, Shao L, et al. Clearance of senescent cells by ABT263 rejuvenates aged hematopoietic stem cells in mice. Nat Med 2016; 22: 78-83

40 Zhu Y, Tchkonia T, Fuhrmann-Stroissnigg H, et al. Identification of a novel senolytic agent, navitoclax, targeting the Bcl-2 family of anti-apoptotic factors. Aging Cell 2016; 15: 428-435.

41 Zhu Y, Tchkonia T, Pirtskhalava T, et al. The Achilles' heel of senescent cells: from transcriptome to senolytic drugs. Aging Cell 2015; 14: 644-658.

42 Baker DJ, Childs BG, Durik M, et al. Naturally occurring p16(Ink4a)-positive cells shorten healthy lifespan. Nature 2016; 530: 184-189.

43 Cui H, Ge J, Xie N, et al. miR-34a promotes fibrosis in aged lungs by inducing alveolar epithelial dysfunctions. Am J Physiol Lung Cell Mol Physiol 2017; 312: L415-L424.

44 Aoshiba K, Tsuji T, Kameyama S, et al. Senescence-associated secretory phenotype in a mouse model of bleomycin-induced lung injury. Exp Toxicol Pathol 2013; 65: 1053-1062.

45 Aoshiba K, Tsuji T, Nagai A. Bleomycin induces cellular senescence in alveolar epithelial cells. Eur Respir J 2003; 22: 436-443.

46 Yanai H, Shteinberg A, Porat Z, et al. Cellular senescence-like features of lung fibroblasts derived from idiopathic pulmonary fibrosis patients. Aging 2015; 7: 664-672.

47 Vaughan AE, Brumwell AN, Xi Y, et al. Lineage-negative progenitors mobilize to regenerate lung epithelium after major injury. Nature 2015; 517: 621-625.

48 Qu P, Du H, Wang X, et al. Matrix metalloproteinase 12 overexpression in lung epithelial cells plays a key role in emphysema to lung bronchioalveolar adenocarcinoma transition. Cancer Res 2009; 69: 7252-7261.

49 Aumiller V, Balsara N, Wilhelm J, et al. WNT/ $\beta$-catenin signaling induces IL-1 $\beta$ expression by alveolar epithelia cells in pulmonary fibrosis. Am J Respir Cell Mol Biol 2013; 49: 96-104.

50 Portnoy J, Curran-Everett D, Mason RJ. Keratinocyte growth factor stimulates alveolar type II cell proliferation through the extracellular signal-regulated kinase and phosphatidylinositol 3-OH kinase pathways. Am J Respir Cell Mol Biol 2004; 30: 901-907.

51 Selman M, Pardo A, Kaminski N. Idiopathic pulmonary fibrosis: aberrant recapitulation of developmental programs? PLoS Med 2008; 5: e62.

52 Alder JK, Chen JJ, Lancaster L, et al. Short telomeres are a risk factor for idiopathic pulmonary fibrosis. Proc Natl Acad Sci USA 2008; 105: 13051-13056.

53 Armanios MY, Chen JJ, Cogan JD, et al. Telomerase mutations in families with idiopathic pulmonary fibrosis. $N$ Engl J Med 2007; 356: 1317-1326.

54 Storer M, Mas A, Robert-Moreno A, et al. Senescence is a developmental mechanism that contributes to embryonic growth and patterning. Cell 2013; 155: 1119-1130.

55 Muñoz-Espín D, Cañamero M, Maraver A, et al. Programmed cell senescence during mammalian embryonic development. Cell 2013; 155: 1104-1118.

56 Liu H, Fergusson MM, Castilho RM, et al. Augmented Wnt signaling in a mammalian model of accelerated aging. Science 2007; 317: 803-806.

57 Chilosi M, Poletti V, Zamò A, et al. Aberrant Wnt/ $\beta$-catenin pathway activation in idiopathic pulmonary fibrosis. Am J Pathol 2003; 162: 1495-1502.

58 Königshoff M, Balsara N, Pfaff EM, et al. Functional Wnt signaling is increased in idiopathic pulmonary fibrosis. PLoS One 2008; 3: e2142.

59 Yang IV, Burch LH, Steele MP, et al. Gene expression profiling of familial and sporadic interstitial pneumonia. Am J Respir Crit Care Med 2007; 175: 45-54.

60 Yilmaz O, Oztay F, Kayalar O. Dasatinib attenuated bleomycin-induced pulmonary fibrosis in mice. Growth Factors 2015; 33: 366-375. 
61 Zhu F, Li Y, Zhang J, et al. Senescent cardiac fibroblast is critical for cardiac fibrosis after myocardial infarction. PLoS One 2013; 8: e74535.

62 Alsafadi HN, Staab-Weijnitz CA, Lehmann M, et al. An ex vivo model to induce early fibrosis-like changes in human precision-cut lung slices. Am J Physiol Lung Cell Mol Physiol 2017; 312: L896-L902. 